\title{
Simple Analytical Expression for Vector Hypernuclear Asymmetry in Nonmesonic Decay of ${ }_{\Lambda}^{5} \mathrm{He}$ and ${ }_{\Lambda}^{12} \mathrm{C}$
}

\author{
César Barbero, \\ Departamento de Física, Facultad de Ciencias Exactas, \\ Universidad Nacional de La Plata, C.C. 67, 1900 La Plata, Argentina. \\ Francisco Krmpotić, \\ Instituto de Física, Universidade de São Paulo, \\ C. P. 66318, 05315-970 São Paulo, SP, Brazil, \\ Instituto de Física La Plata, \\ CONICET, 1900 La Plata, Argentina. \\ and Alfredo P. Galeão \\ Instituto de Física Teórica, Universidade Estadual Paulista, \\ Rua Pamplona 145, 01405-900 São Paulo, SP, Brazil.
}

Received on 18 March, 2006

\begin{abstract}
We present general explicit expressions for a shell-model calculation of the vector hypernuclear parameter in nonmesonic weak decay. We use a widely accepted effective coupling Hamiltonian involving the exchange of the complete pseudoscalar and vector meson octets $\left(\pi, \eta, K, \rho, \omega, K^{*}\right)$. In contrast to the approximated formula widely used in the literature, we correctly treat the contribution of transitions originated from single-proton states beyond the $s$-shell. Exact and simple analytical expressions are obtained for the particular cases of ${ }_{\Lambda}^{5} \mathrm{He}$ and ${ }_{\Lambda}^{12} \mathrm{C}$, within the one-pion-exchange model. Numerical computations of the asymmetry parameter, $a_{\Lambda}$, are presented. Our results show a qualitative agreement with other theoretical estimates but also a contradiction with recent experimental determinations. Our simple analytical formulas provide a guide in searching the origin of such discrepancies, and they will be useful for helping to solve the hypernuclear weak decay puzzle.
\end{abstract}

Keywords: Hypernuclear decay; Asymmetry parameter; One-meson-exchange model

The free decay of a $\Lambda$ hyperon occurs almost exclusively through the mesonic mode, $\Lambda \rightarrow \pi N$, emerging the nucleon with a momentum of about $100 \mathrm{MeV} / \mathrm{c}$. Inside the nuclear medium $\left(p_{F} \sim 270 \mathrm{MeV} / \mathrm{c}\right)$ this mode is Pauli blocked and, for all but the lightest $\Lambda$ hypernuclei $(A \geq 5)$, the weak decay is dominated by the nonmesonic channel, $\Lambda N \rightarrow N N$, with enough kinetic energy to put the two emitted nucleons above the Fermi surface. The nonmesonic hypernuclear weak decay (NMHWD) offers us a very unique opportunity to investigate strangeness-changing weak interaction between hadrons. Assuming that the NMHWD is saturated by the $\Lambda N \rightarrow N N$ mode, the transitions receive contributions either from neutrons $(\Lambda n \rightarrow n n)$ and protons $(\Lambda p \rightarrow n p)$, with rates $\Gamma_{n}$ and $\Gamma_{p}$, respectively $\left(\Gamma_{N M}=\Gamma_{n}+\Gamma_{p}, \Gamma_{n / p}=\Gamma_{n} / \Gamma_{p}\right)$. Over the last three decades amount of theoretical and experimental effort has been invested in solving an interesting puzzle: the theoretical models cannot reproduce simultaneously the experimental values of $\Gamma_{n / p}$ and $a_{\Lambda}$.

From the experimental side there is actually an intense activity, as can be seen in the light of the experiments under way and/or planned at (KEK) [1], (FINUDA) [2] and Brookhaven National Laboratory (BNL) [3]. The preliminary results give a $\Gamma_{n / p}$ ratio value very close to 0.5 [4-6] and the measurements of $a_{\Lambda}$ favor a negative value for ${ }_{\Lambda}^{12} C$ and a positive value for ${ }_{\Lambda}^{5} \mathrm{He}$ [5-9]. On the other hand, from the pioneering work of Block and Dalitz [10] there have been many theoretical attempts dedicated to solving the puzzle. The earlier studies were based on the simplest model of the virtual pion exchange
[11]. This model naturally explains the long range part of the two body interaction, and it reproduces reasonably well the total decay rate but fails badly in reproducing the other observables. In order to achieve a better description, some improvements have been introduced: (i) models that include the exchange of different combinations of other heavier mesons, like $\eta, K, \rho, \omega$ and $K^{*}[12]-[19]$, to reproduce the short range part of the interaction ; (ii) analysis of the two nucleon $(2 \mathrm{~N})$ stimulated process $\Lambda N N \rightarrow N N N$ [20, 21]; (iii) inclusion of interaction terms that violate the isospin $\Delta T=1 / 2$ rule [22]-[24]; (iv) description of the short range baryon-baryon interaction in terms of quark degrees of freedom [25, 26]; (v) correlated (in the form of $\sigma$ and $\rho$ mesons) and uncorrelated two-pion exchanges [27]-[31]. We emphasize that none of these models give a fully satisfactory description of all the NMHWD observables simultaneously, even though a consistent (though not sufficient) increase of $\Gamma_{n / p}$ has been found.

All such models reproduce quite well $\Gamma_{n m}$, but seem to strongly underestimate the experimental $n / p$ branching ratio, $\Gamma_{n} / \Gamma_{p}$. Besides, the measurements favor a negative value of $a_{\Lambda}$ for ${ }_{\Lambda}^{12} \mathrm{C}$ and a positive one for ${ }_{\Lambda}^{5} \mathrm{He}$, meanwhile all existing calculations based on strict one-meson-exchange (OME) models [14-16, 18, 19, 26, 32, 33] find values between -0.73 and -0.19 for ${ }_{\Lambda}^{5} \mathrm{He}[34,35]$ and, when results are available in the same model, very similar values for ${ }_{\Lambda}^{12} C$.

Most calculations of the asymmetry parameter make use of an approximate formula (Eq. (18), below) which, however, is valid only for $s$-shell hypernuclei. Since an essential aspect 


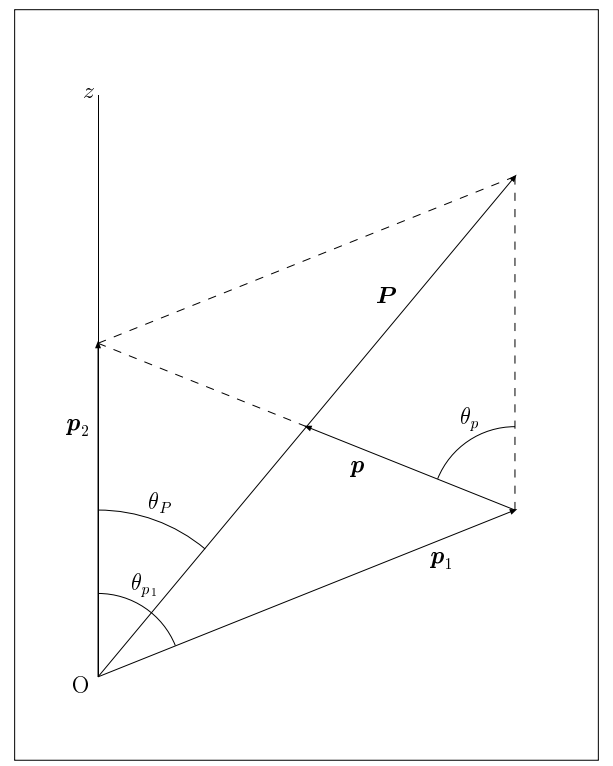

FIG. 1: Coordinate system for the calculation of decay strengths.

in the asymmetry puzzle presented above concerns the comparison of its values for ${ }_{\Lambda}^{5} \mathrm{He}$ and ${ }_{\Lambda}^{12} \mathrm{C}$, it would be of great interest to have an expression that is applicable to both cases. Besides, simple formulas containing kinematical factors and nuclear matrix elements separately, will provide a manageable and useful tool. They will be a guide in searching the origin of discrepancies between theoretical calculations and experimental data.

Thus, we present in this paper a general formalism for the vector asymmetry parameter in nonmesonic decay and derive exact and simple analytical expressions, within the simplest one-pion exchange model (OPEM), for the particular cases of ${ }_{\Lambda}^{5} \mathrm{He}$ and ${ }_{\Lambda}^{12} \mathrm{C}$ hypernuclei.

Single- $\Lambda$ hypernuclei produced in a $\left(\pi^{+}, K^{+}\right)$reaction end up with considerable vector polarization along the direction normal to the reaction plane, $\hat{\boldsymbol{n}}=\left(\boldsymbol{p}_{\pi^{+}} \times \boldsymbol{p}_{K^{+}}\right) /\left|\boldsymbol{p}_{\pi^{+}} \times \boldsymbol{p}_{K^{+}}\right|$. The angular distribution of protons emitted in $\Lambda p \rightarrow n p$ decay is given by [34]

$$
\frac{d \Gamma\left(J_{I} M_{I} \rightarrow \hat{\boldsymbol{p}}_{2} t_{p}\right)}{d \Omega_{p_{2}}}=\frac{\Gamma_{p}}{4 \pi}\left(1+P_{V} A_{V} \hat{\boldsymbol{p}}_{2} \cdot \hat{\boldsymbol{n}}\right),
$$

where $\Gamma_{p}$ is the full proton-induced decay rate, and

$$
A_{V}=3 \sqrt{\frac{J_{I}}{J_{I}+1}} \frac{\sigma_{1}\left(J_{I}\right)}{\sigma_{0}\left(J_{I}\right)}
$$

is the vector hypernuclear asymmetry, with

$$
\begin{aligned}
& \sigma_{0}\left(J_{I}\right)=\sum_{M_{I}} \sigma\left(J_{I} M_{I}\right) \\
& \sigma_{1}\left(J_{I}\right)=\frac{1}{\sqrt{J_{I}\left(J_{I}+1\right)}} \sum_{M_{I}} M_{I} \sigma\left(J_{I} M_{I}\right),
\end{aligned}
$$

being

$$
\sigma\left(J_{I} M_{I}\right)=\int d \Omega_{p_{1}} \int d F \sum_{s_{1} s_{2} M_{F}}\left|\left\langle\boldsymbol{p}_{1} s_{1} t_{n} \boldsymbol{p}_{2} s_{2} t_{p} J_{F} M_{F}|V| J_{I} M_{I}\right\rangle_{\mathcal{A}}\right|^{2}
$$

Here $V$ is the OME potential involving the complete pseudoscalar and vector meson octets $\left(\pi, \eta, K, \rho, \omega, K^{*}\right)$ which has been extensively discussed in Refs. [17, 18], $\boldsymbol{p}_{1} s_{1}\left(\boldsymbol{p}_{2} s_{2}\right)$ are the momenta and spin projections of the emitted neutron (proton) and

$$
\int d F \ldots=2 \pi \sum_{J_{F}} \int \frac{p_{2}^{2} d p_{2}}{(2 \pi)^{3}} \int \frac{p_{1}^{2} d p_{1}}{(2 \pi)^{3}} \delta\left(\frac{p_{1}^{2}}{2 \mathrm{M}}+\frac{p_{2}^{2}}{2 \mathrm{M}}+\frac{\left|\boldsymbol{p}_{1}+\boldsymbol{p}_{2}\right|^{2}}{2 \mathrm{M}_{F}}-\Delta_{J_{F}}\right) \ldots
$$

with $\mathrm{M}$ and $\mathrm{M}_{F}$ being the nucleon and residual nucleus masses and $\Delta_{J_{F}}$ the liberated energy.

We assume the hyperon to stay in the $\mathrm{j}_{\Lambda}=1 s_{1 / 2}$ single-particle state, and the initial hypernuclear state is built as $\left|J_{I}\right\rangle \equiv$ $\left|\left(\mathrm{j}_{\Lambda} J_{C}\right) J_{I}\right\rangle$, where $\left|J_{C}\right\rangle$ is the ${ }^{A-1} Z$ ground state. Thus, the intrinsic asymmetry parameter is defined as

$$
a_{\Lambda}=\left\{\begin{array}{cl}
A_{V} & \text { for } J_{I}=J_{C}+1 / 2, \\
-\frac{J_{I}+1}{J_{I}} A_{V} & \text { for } J_{I}=J_{C}-1 / 2 .
\end{array}\right.
$$

Rewriting the transition amplitude in (4) in the total spin $\left(S, M_{S}\right)$ and isospin $\left(T, M_{T}\right)$ basis we get

$$
\sigma\left(J_{I} M_{I}\right)=\int d \Omega_{p_{1}} \int d F \sum_{S M_{S} M_{F}}\left|\sum_{T}(-)^{T}\left\langle p \boldsymbol{P S} M_{S} T J_{F} M_{F}|V| J_{I} M_{I}\right\rangle_{\mathcal{A}}\right|^{2},
$$

where $\boldsymbol{p}=\frac{1}{2}\left(\boldsymbol{p}_{2}-\boldsymbol{p}_{1}\right), \boldsymbol{P}=\boldsymbol{p}_{1}+\boldsymbol{p}_{2}$ and

$$
\begin{aligned}
& \left\langle p \operatorname{PSM}_{S} T J_{F} M_{F}|V| J_{I} M_{I}\right\rangle_{\mathcal{A}}
\end{aligned}
$$

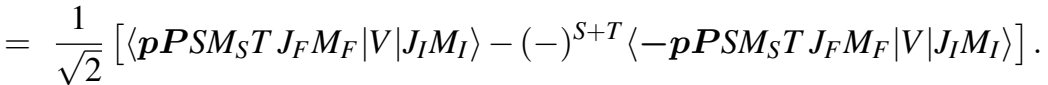


For the integration in (7) we take the $z$-axis as indicated in Fig. 1 and choose $p_{2}, \theta_{p_{1}}$ and $\phi_{p_{1}}$ as independent variables. Thus, expanding the final state in terms of relative and center-of-mass partial waves of the emitted nucleons [17], after integration on $\phi_{p_{1}}$ and recoupling of angular momentum, we get

$$
\begin{aligned}
\sigma\left(J_{I} M_{I}\right) & =\frac{1}{2}(4 \pi)^{4} \int d \cos \theta_{p_{1}} \int d F \sum_{S T T^{\prime}}(-)^{T+T^{\prime}} \\
& \times \sum_{l L \lambda J} \sum_{l^{\prime} L^{\prime} \lambda^{\prime} J^{\prime}} i^{-l^{\prime}-L^{\prime}-l-L}(-)^{\lambda+S+J+J^{\prime}+J_{I}+J_{F}} \hat{l} \hat{l}^{\prime} \hat{L} \hat{L^{\prime}} \hat{\lambda} \hat{\lambda}^{\prime} \hat{J} \hat{J}^{\prime} \\
& \times \sum_{k K \kappa} \hat{\kappa} \hat{J}_{I}\left(J_{I} M_{I} \kappa 0 \mid J_{I} M_{I}\right)\left(l 0 l^{\prime} 0 \mid k 0\right)\left(L 0 L^{\prime} 0 \mid K 0\right)\left[Y_{k}\left(\theta_{p}, \pi\right) \otimes Y_{K}\left(\theta_{P}, 0\right)\right]_{\kappa 0} \\
& \times\left\{\begin{array}{ccc}
J_{I} & \kappa & J_{I} \\
J & J_{F} & J^{\prime}
\end{array}\right\}\left\{\begin{array}{ccc}
\kappa & J^{\prime} & J \\
S & \lambda & \lambda^{\prime}
\end{array}\right\}\left\{\begin{array}{ccc}
l & l^{\prime} & k \\
L & L^{\prime} & K \\
\lambda & \lambda^{\prime} & \kappa
\end{array}\right\} \\
& \times\left(p l P L \lambda S J T J_{F} ; J_{I}|V| J_{I}\right)\left(p l^{\prime} P L^{\prime} \lambda^{\prime} S J^{\prime} T^{\prime} J_{F} ; J_{I}|V| J_{I}\right)^{*} .
\end{aligned}
$$

where $\left(p l P L \lambda S J T J_{F} ; J_{I}|V| J_{I}\right)=\frac{1}{\sqrt{2}}\left[1-(-)^{l+S+T}\right]\left\langle p l P L \lambda S J T J_{F} ; J_{I}|V| J_{I}\right\rangle$.

From [17] we have

$$
\begin{aligned}
& \left\langle p l P L \lambda S J T J_{F} ; J_{I}|V| J_{I}\right\rangle \\
& =(-)^{J_{F}+J-J_{I}} \hat{J}_{I}^{-1} \sum_{\mathrm{j}_{p}}\left\langle J_{I}||\left(a_{\mathrm{j}_{\Lambda}}^{\dagger} a_{\mathrm{j}_{p}}^{\dagger}\right)_{J} \| J_{F}\right\rangle \mathcal{M}\left(p l P L \lambda S J T ; \mathrm{j}_{\Lambda} \mathrm{j}_{p}\right),
\end{aligned}
$$

where

$$
\mathcal{M}\left(p l P L \lambda S J T ; \mathrm{j}_{\Lambda} \mathrm{j}_{p}\right)=\frac{1}{\sqrt{2}}\left[1-(-)^{l+S+T}\right]\left(p l P L \lambda S J T|V| \mathrm{j}_{\Lambda} \mathrm{j}_{p} J\right)
$$

The two-particle spectroscopic amplitudes are cast as [17]

$$
\left\langle J_{I}\left\|\left(a_{\mathrm{j}_{\Lambda}}^{\dagger} a_{\mathrm{j}_{p}}^{\dagger}\right)_{J}\right\| J_{F}\right\rangle=(-)^{J+J_{I}+J_{F}} \hat{J J_{I}}\left\{\begin{array}{ccc}
J_{C} & J_{I} & j_{\Lambda} \\
J & j_{p} & J_{F}
\end{array}\right\}\left\langle J_{C}\left\|a_{\mathrm{j}_{p}}^{\dagger}\right\| J_{F}\right\rangle .
$$

To continue, we will adopt the extreme shell model and restrict our attention to cases where the single-proton states are completely filled in $\left|J_{C}\right\rangle$. This is so, for the cores of both ${ }_{\Lambda}^{5} \mathrm{He}$ $\left(J_{I}=1 / 2, J_{C}=0\right)$, and ${ }_{\Lambda}^{12} \mathrm{C}\left(J_{I}=1, J_{C}=3 / 2\right)$ [36].

In such cases, the final nuclear states take the form $\left|J_{F}\right\rangle \equiv$ $\left|\left(\mathrm{j}_{p}^{-1} J_{C}\right) J_{F}\right\rangle$. This leads to

$$
\left\langle J_{C}|| a_{j_{p}}^{\dagger}|| J_{F}\right\rangle=(-)^{J_{F}+J_{C}+j_{p}} \hat{J}_{F} .
$$

$$
\begin{aligned}
\sigma_{\kappa}\left(J_{I}\right) & =\frac{4}{\pi} \hat{J}_{I}^{3} \hat{\kappa}^{-1} \sum_{\mathrm{j}_{p}} \int d \cos \theta_{p_{1}} \int d F_{\mathrm{j}_{p}} \sum_{S T T^{\prime}}(-)^{T+T^{\prime}} \\
& \times \sum_{l L \lambda J} \sum_{l^{\prime} L^{\prime} \lambda^{\prime} J^{\prime}} i^{-l^{\prime}-L^{\prime}-l-L}(-)^{\lambda+S+J_{I}+J_{C}-j_{p}+\kappa} \hat{l} \hat{l} \hat{L} \hat{L} \hat{L^{\prime}} \hat{\lambda} \hat{\lambda}^{\prime} \hat{J}^{2} \hat{J}^{2} \\
& \times \sum_{k K}\left(l 0 l^{\prime} 0 \mid k 0\right)\left(L 0 L^{\prime} 0 \mid K 0\right)\left[Y_{k}\left(\theta_{p}, \pi\right) \otimes Y_{K}\left(\theta_{P}, 0\right)\right]_{\kappa 0} \\
& \times\left\{\begin{array}{ccc}
j_{\Lambda} & J_{I} & J_{C} \\
J_{I} & j_{\Lambda} & \kappa
\end{array}\right\}\left\{\begin{array}{ccc}
\kappa & j_{\Lambda} & j_{\Lambda} \\
j_{p} & J & J^{\prime}
\end{array}\right\}\left\{\begin{array}{ccc}
\kappa & J^{\prime} & J \\
S & \lambda & \lambda^{\prime}
\end{array}\right\}\left\{\begin{array}{ccc}
l & l^{\prime} & k \\
L & L^{\prime} & K \\
\lambda & \lambda^{\prime} & \kappa
\end{array}\right\} \\
& \times \mathcal{M}\left(p l P L \lambda S J T ; \mathrm{j}_{\Lambda} \mathrm{j}_{p}\right) \mathcal{M}^{*}\left(p l^{\prime} P L^{\prime} \lambda^{\prime} S J^{\prime} T^{\prime} ; j_{\Lambda} \mathrm{j}_{p}\right),
\end{aligned}
$$


where

$$
\int d F_{\mathrm{j}_{p}} \ldots=\int p_{2}^{2} d p_{2} \int p_{1}^{2} d p_{1} \delta\left(\frac{p_{1}^{2}}{2 \mathrm{M}}+\frac{p_{2}^{2}}{2 \mathrm{M}}+\frac{\left|\boldsymbol{p}_{1}+\boldsymbol{p}_{2}\right|^{2}}{2 \mathrm{M}_{F}}-\Delta_{\mathrm{j}_{p}}\right) \ldots
$$

The intrinsic asymmetry parameter can finally be written as [31]

$$
a_{\Lambda}=\frac{\omega_{1}}{\omega_{0}}
$$

where

$$
\begin{aligned}
\omega_{\kappa} & =(-)^{\kappa} \frac{8}{\sqrt{2 \pi}} \hat{\kappa}^{-1} \sum_{\mathrm{j}_{p}} \int d \cos \theta_{p_{1}} \int d F_{\mathrm{j}_{p}} Y_{\kappa}\left(\theta_{p}, 0\right) \\
& \times \sum_{T T^{\prime}}(-)^{T+T^{\prime}} \sum_{L S} \sum_{l \lambda J} \sum_{l^{\prime} \lambda^{\prime} J^{\prime}} i^{l-l^{\prime}}(-)^{\lambda+\lambda^{\prime}+S+L+j_{p}+\frac{1}{2}} \\
& \times \hat{l} \hat{l}^{\prime} \hat{\lambda} \hat{\lambda}^{\prime} \hat{J}^{2} \hat{J}^{\prime 2}\left(l 0 l^{\prime} 0 \mid \kappa 0\right) \\
& \times\left\{\begin{array}{ccc}
\kappa & 1 / 2 & 1 / 2 \\
j_{p} & J & J^{\prime}
\end{array}\right\}\left\{\begin{array}{lll}
\kappa & J^{\prime} & J \\
S & \lambda & \lambda^{\prime}
\end{array}\right\}\left\{\begin{array}{ccc}
l^{\prime} & l & \kappa \\
\lambda & \lambda^{\prime} & L
\end{array}\right\} \\
& \times \mathcal{M}\left(p l P L \lambda S J T ; \mathrm{j}_{\Lambda} \mathrm{j}_{p}\right) \mathcal{M}^{*}\left(p l^{\prime} P L \lambda^{\prime} S J^{\prime} T^{\prime} ; \mathrm{j}_{\Lambda} \mathrm{j}_{p}\right),(17),
\end{aligned}
$$

with $L=0$ for the $1 s_{1 / 2}$ state, and $L=0$ and 1 for the $1 p_{3 / 2}$ state. It has been verified that $\omega_{0}=\Gamma_{p}$, in such way that the new information is carried by $\omega_{1}$.
The presence of the Clebsch-Gordan coefficient in Eq. (17), for $\kappa=1$, ensures that $l$ and $l^{\prime}$ have opposite parities. Since the initial state in the two matrix elements has a definite parity, this implies that all contributions to $\omega_{1}$ come from interference terms between the parity-conserving and the parityviolating parts of the transition potential. Furthermore, the antisymmetrization factor in Eq. (11) shows that the two final states have $T \neq T^{\prime}$. These are general properties of the asymmetry parameter.

We have used these general expressions for the numerical computation of $a_{\Lambda}$ within several OME models. The values of $\omega_{0}$ and $\omega_{1}$ are shown in Tables I and II, for ${ }_{\Lambda}^{5} \mathrm{He}$ and ${ }_{\Lambda}^{12} \mathrm{C}$ respectively, in units of the free $\Lambda$ decay constant, $\Gamma_{\Lambda}^{(0)}=2.50 \times 10^{-6} \mathrm{eV}$. In the case of ${ }_{\Lambda}^{5} \mathrm{He}$, we have also included, between parentheses, the values obtained with the approximate formula usually adopted in the literature [34], which is strictly valid only for $s$-shell hypernuclei:

$$
A_{V}\left({ }_{\Lambda}^{5} \mathrm{He}\right) \approx \frac{2 \Re\left[\sqrt{3} a e^{*}-b\left(c^{*}-\sqrt{2} d^{*}\right)+\sqrt{3} f\left(\sqrt{2} c^{*}+d^{*}\right)\right]}{|a|^{2}+|b|^{2}+3\left(|c|^{2}+|d|^{2}+|e|^{2}+|f|^{2}\right)},
$$

where

$$
\begin{array}{lll}
a=\left\langle n p,{ }^{1} \mathrm{~S}_{0}|V| \Lambda p,{ }^{1} \mathrm{~S}_{0}\right\rangle, & b=i\left\langle n p,{ }^{3} \mathrm{P}_{0}|V| \Lambda p,{ }^{1} \mathrm{~S}_{0}\right\rangle, & c=\left\langle n p,{ }^{3} \mathrm{~S}_{1}|V| \Lambda p,{ }^{3} \mathrm{~S}_{1}\right\rangle \\
d=-\left\langle n p,{ }^{3} \mathrm{D}_{1}|V| \Lambda p,{ }^{3} \mathrm{~S}_{1}\right\rangle, & e=i\left\langle n p,{ }^{1} \mathrm{P}_{1}|V| \Lambda p,{ }^{3} \mathrm{~S}_{1}\right\rangle, & f=-i\left\langle n p,{ }^{3} \mathrm{P}_{1}|V| \Lambda p,{ }^{3} \mathrm{~S}_{1}\right\rangle
\end{array}
$$

We find values ranging from -0.62 to -0.29 , in qualitative agreement with other theoretical estimates [14-16, 18, 19, 26, $32-35]$ but in contradiction with some recent experimental determinations [5-9].

We remark here that the approximate formula ignores the fact that the final state of nonmesonic decay is a three-body one and does not include the full contribution of the transitions coming from proton states beyond the $s$-shell, being therefore of only limited validity, and should not be used for $p$-shell hypernuclei such as ${ }_{\Lambda}^{12} \mathrm{C}$, or, even worse, for heavier ones. This being said, comparison of the corresponding values for $a_{\Lambda}$ in Table I shows that the formula works well within its range of validity.

Also, from Table II we observe that the $p$-shell contribu- tions to $\omega_{0}$ and $\omega_{1}$ for ${ }_{\Lambda}^{12} \mathrm{C}$ are by no means negligible. However, they are also in approximately the same ratio, so that the effect on $a_{\Lambda}$ is much smaller.

In order to obtain simple and manageable analytical expressions for the asymmetry parameter, we adopt now the simplest OME model, which takes into account only the contribution of one pion exchange. As can be seen form our numerical results, the main characteristics of the nonmesonic hypernuclear weak decay asymmetry are well described within this OPEM. Then, as a starting point, this model will be enough to obtain such analytical formula[37].

After a straightforward analytical evaluation of the summation terms indicated in (17), together with the calculation of the nuclear matrix elements indicated in Eqs. (11)-(13) within 
TABLE I: Results for the asymmetry parameter, $a_{\Lambda}$, for nonmesonic decay of ${ }_{\Lambda}^{5} \mathrm{He}$.

\begin{tabular}{|c|ccc|}
\hline \hline Model/Calculations & $\omega_{0}\left(1 s_{1 / 2}\right)$ & $\omega_{1}\left(1 s_{1 / 2}\right)$ & $a_{\Lambda}$ \\
\hline$\pi$ & 0.6492 & -0.2913 & $-0.4487(-0.4456)$ \\
$(\pi, \eta, K)$ & 0.3920 & -0.2412 & $-0.6153(-0.6384)$ \\
$\pi+\rho$ & 0.5937 & -0.1776 & $-0.2991(-0.3155)$ \\
$(\pi, \eta, K)+\left(\rho, \omega, K^{*}\right)$ & 0.5526 & -0.2974 & $-0.5382(-0.5388)$ \\
\hline Experiment & KEK-PS E278 [9] & $0.24 \pm 0.22$ \\
$a_{\Lambda}=A_{V}\left({ }_{\Lambda}^{5} \mathrm{He}\right)$ & KEK-PS E462 [7] (preliminary) & $0.11 \pm 0.08 \pm 0.04$ \\
\hline
\end{tabular}

TABLE II: Results for the asymmetry parameter, $a_{\Lambda}$, for nonmesonic decay of ${ }_{\Lambda}^{12} \mathrm{C}$.

\begin{tabular}{|c|ccccc|}
\hline \hline Model/Calculations & $\omega_{0}\left(1 s_{1 / 2}\right)$ & $\omega_{0}\left(1 p_{3 / 2}\right)$ & $\omega_{1}\left(1 s_{1 / 2}\right)$ & $\omega_{1}\left(1 p_{3 / 2}\right)$ & $a_{\Lambda}$ \\
\hline$\pi$ & 0.5206 & 0.5954 & -0.2400 & -0.2596 & -0.4477 \\
$(\pi, \eta, K)$ & 0.3336 & 0.3817 & -0.2057 & -0.2217 & -0.5975 \\
$\pi+\rho$ & 0.4922 & 0.5514 & -0.1461 & -0.1596 & -0.2930 \\
$(\pi, \eta, K)+\left(\rho, \omega, K^{*}\right)$ & 0.4619 & 0.5083 & -0.2546 & -0.2599 & -0.5303 \\
\hline Experiment & KEK-PS E160 [8] & & $-0.9 \pm 0.3$ \\
$a_{\Lambda}=-2 A_{V}\left({ }_{\Lambda}^{12} \mathrm{C}\right)$ & KEK-PS E508 [7] (preliminary) & & $-0.44 \pm 0.32$ \\
\hline \hline
\end{tabular}

the strict OPEM, we can write

$$
\begin{aligned}
& \omega_{0}=\frac{24}{\pi} \int d \cos \theta_{p_{1}} \int d F_{\mathrm{j}_{p}}\left[3\left(\mathrm{~S}_{\pi}^{0}\right)^{2}+18\left(\mathrm{~T}_{\pi}^{20}\right)^{2}+\left(\mathrm{P}_{\pi}^{10}\right)^{2}\right] \\
& \omega_{1}=\frac{32 \sqrt{3}}{\sqrt{\pi}} \int d \cos \theta_{p_{1}} \int d F_{\mathrm{j}_{p}} Y_{10}\left(\theta_{p}, 0\right)\left(\mathrm{S}_{\pi}^{0}-2 \mathrm{~T}_{\pi}^{20}\right) \mathrm{P}_{\pi}^{10}
\end{aligned}
$$

for ${ }_{\Lambda}^{5} \mathrm{He}$, and

$$
\begin{aligned}
\omega_{0} & =\frac{24}{\pi} \int d \cos \theta_{p_{1}} \int d F_{\mathrm{j}_{p}}\left\{(1+R)\left[3\left(\mathrm{~S}_{\pi}^{0}\right)^{2}+18\left(\mathrm{~T}_{\pi}^{20}\right)^{2}+\left(\mathrm{P}_{\pi}^{10}\right)^{2}\right]\right. \\
& \left.+7\left(\mathrm{~S}_{\pi}^{1}\right)^{2}+\frac{1}{5}\left(\mathrm{~T}_{\pi}^{11}\right)^{2}+\frac{9}{5}\left(\mathrm{~T}_{\pi}^{31}\right)^{2}+\frac{7}{3}\left(\mathrm{P}_{\pi}^{21}\right)^{2}\right\} \\
\omega_{1} & =\frac{32 \sqrt{3}}{\sqrt{\pi}} \int d \cos \theta_{p_{1}} \int d F_{\mathrm{j}_{p}} Y_{10}\left(\theta_{p}, 0\right)\left[(1+R)\left(\mathrm{S}_{\pi}^{0}-2 \mathrm{~T}_{\pi}^{20}\right) \mathrm{P}_{\pi}^{10}\right. \\
& \left.+\left(\mathrm{S}_{\pi}^{1}+\frac{1}{5} \mathrm{~T}_{\pi}^{11}-\frac{9}{5} \mathrm{~T}_{\pi}^{31}\right) \mathrm{P}_{\pi}^{21}\right]
\end{aligned}
$$

for ${ }_{\Lambda}^{12} \mathrm{C}$. Here $\mathrm{S}_{\pi}^{l}, \mathrm{~T}_{\pi}^{l l}$ and $\mathrm{P}_{\pi}^{l l}$, and $R=(b P)^{2} / 3$ are, respectively, the nuclear moments and the ratio between the $1 p_{3 / 2}$ and $1 s_{1 / 2}$ contributions defined in Section IV C from Ref. [17]. These formulas clearly show that the $s$-part of ${ }_{\Lambda}^{12} \mathrm{C}$ results agrees with the ${ }_{\Lambda}^{5} \mathrm{He}$ ones. This has also been tested numerically, as can be seen from Tables I and II. There we observe that this conclusion is also valid within the complete OME model. Simultaneously, our numerical computation shows that the contribution of the $p$-shell of ${ }_{\Lambda}^{12} \mathrm{C}$ has approximately the same magnitude that the $s$-one, both for $\omega_{0}$ and $\omega_{1}$ (this has been extensively discussed in Ref. [17] concerning the decay rate).

On the other side, it is also well known that the main con- tribution comes from the lower quantum numbers and, as it has been discussed in Ref. [17], the tensorial $T_{\pi}^{20}$ term is the dominant one in the hypernuclear weak decay case. Thus, approximating the $p$-shell contribution of ${ }_{\Lambda}^{12} \mathrm{C}$ by the $s$-one, and retaining only the terms containing the tensorial moment $\mathrm{T}_{\pi}^{20}$ in our formula, we arrive to a very simple expression for $a_{\Lambda}$ :

$$
a_{\Lambda} \simeq-\frac{4 \sqrt{3 \pi}}{27} \frac{\int d \cos \theta_{p_{1}} \int d F_{\mathrm{j} p} Y_{10}\left(\theta_{p}, 0\right) \mathrm{T}_{\pi}^{20} \mathrm{P}_{\pi}^{10}}{\int d \cos \theta_{p_{1}} \int d F_{\mathrm{j}_{p}}\left(\mathrm{~T}_{\pi}^{20}\right)^{2}}
$$

This expression is valid both for ${ }_{\Lambda}^{5} \mathrm{He}$ and $\mathrm{C}_{\Lambda}^{12} \mathrm{C}$ hypernuclear decays, within the OPEM. Our results presented in Eqs. (20), (21) and (22) suggest that, in order to obtain a stronger theo- 
retical dependence of $a_{\Lambda}$ on the particular hypernucleus considered (as indicated by experimental data) future improvements of the model are required.

We believe that a different decay mechanism should be introduced for $s$ - and $p$-shell hypernuclei, beyond the Born approximation. $\mathrm{X}$

Summarizing, we have derived simple formulas for the evaluation of the asymmetry parameter, which exactly include the effects of three-body kinematics in the final states and correctly treat the contribution of transitions originated from proton states beyond the $s$-shell.

Besides, we have deduced exact analytical expressions for the particular cases of ${ }_{\Lambda}^{5} \mathrm{He}$ and ${ }_{\Lambda}^{12} \mathrm{C}$ written, within the OPEM, in terms of nuclear moments.
The numerical values of $a_{\Lambda}$, calculated within different OME models, variate from -0.62 to -0.29 .

The negative value systematically obtained for $a_{\Lambda}$ for the two hypernuclei indicates that it will be hard to get a positive or zero value for it in the ${ }_{\Lambda}^{5} \mathrm{He}$ case, at least within strict OME models. The puzzle posed by the experimental results for $a_{\Lambda}$ in $s$ - and $p$-shell hypernuclei remains unexplained.

However, our results suggest that additional changes in the nuclear model and/or the decay mechanism should be introduced for $s$ - and $p$-shell hypernuclei, in such way that the improvements lead to a stronger theoretical dependence of $a_{\Lambda}$ on the hypernucleus considered, which could reverse the asymmetry sign in the case of ${ }_{\Lambda}^{5} \mathrm{He}$, as required to reproduce the more recent experimental data on this observable.
[1] H. Outa et al., KEK Report No. KEK-PS E462, 2000.

[2] A. Feliciello, Nucl. Phys. A691, 170c (2001); P. Gionatti, Nucl. Phys. A691, 483c (2001).

[3] R.L. Gill, Nucl. Phys. A691, 180c (2001).

[4] G. Garbarino, A. Parreño, and A. Ramos, Phys. Rev. C 69, 054603 (2004); ibid, Nucl. Phys. A754, 137c (2005).

[5] H. Bhang et al., Nucl. Phys. A754, 144c (2005).

[6] H. Outa et al., Nucl. Phys. A754, 157c (2005).

[7] T. Maruta et al., nucl-ex/0402017; ibid, Nucl. Phys. A754, 168c (2005).

[8] S. Ajimura et al., Phys. Lett. B282, 293 (1992).

[9] S. Ajimura et al., Phys. Rev. Lett. 84, 4052 (2000).

[10] M.M. Block and Dalitz, Phys. Rev. Lett. 11, 96 (1963).

[11] J. B. Adams, Phys. Rev. 156, 832 (1967) (Cf. correction pointed out in Ref. [12].)

[12] B. H. J. McKellar and B. F. Gibson, Phys. Rev. C 30, (1984) 322.

[13] K. Takeuchi, H. Takaki, and H. Bandō, Prog. Theor. Phys. 73, 841 (1985).

[14] J. F. Dubach, G. B. Feldman, B. R. Holstein, and L. de la Torre, Ann. Phys. (N.Y.) 249, 146 (1996).

[15] A. Parreño, A. Ramos, and C. Bennhold, Phys. Rev. C 56, 339 (1997) and references therein.

[16] A. Parreño and A. Ramos, Phys. Rev. C 65, 015204 (2001).

[17] C. Barbero, D. Horvat, F. Krmpotić, T. T. S. Kuo, Z. Narančić, and D. Tadić, Phys. Rev. C 66, 055209 (2002).

[18] C. Barbero, C. de Conti, A.P. Galeão, and F. Krmpotić, Nucl. Phys. A726, 267 (2003).

[19] C. Barbero, A.P. Galeão, and F. Krmpotić, Phys. Rev. C 72, 035210 (2005).

[20] A. Ramos, E. Oset, and L. L. Salcedo, Phys. Rev. C50, 2314 (1995).

[21] A. Ramos, M.J. Vicente-Vacas, and E. Oset, Phys. Rev. C55, 735 (1997), Erratum-ibid Phys. Rev. C66, 039903 (2002).
[22] A. Parreño, A. Ramos, C. Bennhold, and K. Maltman, Phys. Lett. B 435, 1 (1998).

[23] W. M. Alberico and G. Garbarino, Phys. Lett. B486, 362 (2000).

[24] J-H. Jun, Phys. Rev. C63, 044012 (2001).

[25] T. Inoue, M. Oka, T. Motoba, and K. Itonaga, Nucl. Phys. A633, 312 (1998)

[26] K. Sasaki, T. Inoue, and M. Oka, Nucl. Phys. A669, 331 (2000), Erratum-ibid A678, 455 (2000).

[27] M. Shmatikov, Phys. Lett. B322, 311 (1994); ibid, Nucl. Phys. A580, 538 (1994).

[28] K. Itonaga, T. Ueda, and T. Motoba, Nucl. Phys. A577, 301c; (1994) ibid, Nucl. Phys. A585, 331c (1995); ibid, Nucl. Phys. A639, 329c (1998).

[29] E. Jido, E. Oset, and J.A. Palomar, Nucl. Phys. A694, 525 (2001).

[30] K. Sasaki, M. Izaki, and M. Oka, Phys. Rev. C71, 035502 (2005).

[31] C. Barbero and A. Mariano, Phys. Rev. C73, 024309 (2006).

[32] K. Itonaga, T. Ueda, and T. Motoba, Phys. Rev. C65, 034617 (2002); K. Itonaga, T. Motoba, and T. Ueda, Electrophoto Production of Strangeness on Nuand Nuclei

(Sendai03), K. Maeda, H. Tamura, S.N. Nakamura, and O. Hashimoto eds., World Scientific (2004) pp. 397-402.

[33] K. Sasaki, T. Inoue, and M. Oka, Nucl. Phys. A702, 477 (2002).

[34] W. M. Alberico and G. Garbarino, Phys. Rep. 369, 1 (2002).

[35] W. M. Alberico, G. Garbarino, A. Parreño, and A. Ramos, Phys. Rev. Lett. 94, 1 (2005).

[36] The decay of the ${ }_{\Lambda}^{11} \mathrm{~B}$ hypernuclei will be analyzed in a forthcoming paper.

[37] An analytical expression including the contribution of the complete octets meson will be deduced and analyzed in a forthcoming paper. 